AN. MED. InTERNA (Madrid) Vol. 19, N. $^{\circ} 7$, pp. $357-360,2002$

\title{
Hipoplasia pulmonar en el adulto: descripción, patogenia y revisión
}

\author{
L. FÁCILA RUBIO*, F. CARRIÓN VALERO, M. GONZÁLEZ MARTÍNEZ, J. MARÍN \\ PARDO
}

Servicios de Neumología y *Cardiología. Hospital Clínico Universitario de Valencia. Universitat de València

LUNG HYPOPLASIA IN THE ADULT: DESCRIPTION, PATHOGENE SIS AND REVIEW

\begin{abstract}
RESUMEN
La hipoplasia pulmonar es una entidad muy poco frecuente caracterizada por una detención en el desarrollo pulmonar, que produce que estos órganos se encuentren poco desarrollados. Habitualmente esta enfermedad es diagnosticada en la infancia y son muy pocos los casos que pasan desapercibidos detectándose en la edad adulta (>18 años).

Presentamos cuatro casos de pacientes adultos diagnosticados de hipoplasia pulmonar por diversas técnicas (de imagen o invasivas), que consultaron por sintomatología inespecífica, sobre todo relacionada con infecciones respiratorias y su posterior evolución a lo largo de los años. Repasamos además la patogenia, etiología y malformaciones asociadas de esta enfermedad así como hacemos una revisión de los trabajos publicados de ésta en este grupo de edad.
\end{abstract}

PALABRAS CLAVE: Hipoplasia pulmonar. Patogenia. Adulto. Malformaciones.

\begin{abstract}
The lung hypoplasia is a very few frequent entity characterized by a detention in the lung development that leads to a poor development of this organ. Habitually this disease is diagnosed in the childood and there are very few cases that pass unknown, being detected in the mature age (>18 years).

We present four cases of diagnoses adult patients of lung hypoplasia for diverse test (imaging or invasive) that consulted for not specific symptoms, mostly related with infections of the lung and their later evo lution over the years. We also review the pathogenesis, etiology and mal formations associated of this disease as well as a review of the published articles in this age group.
\end{abstract}

KEY WORDS: Lung hypoplasia. Pathogenesis. Adult. Malformations.

Fácila Rubio L, Carrión Valero F, González Martínez M, Marín Pardo J. Hipoplasia pulmonar en el adulto: descripción, patogenia y revisión. An Med Interna (Madrid) 2002; 19: 357-360.

\section{INTRODUCCIÓN}

La hipoplasia pulmonar es una anomalía congénita del desarrollo pulmonar muy poco frecuente, que habitualmente se diagnostica en la infancia y tiene una alta mortalidad, de forma que aparece en el $10 \%$ de las necropsias postnatales. Sin embargo, la presencia de esta anomalía en el adulto es excepcional $(1,2)$ ya que lo más probable es que los individuos fallezcan antes de la edad adulta, como consecuencia de infecciones pulmonares intercurrentes o de otras anomalías congénitas asociadas (3-5). En la revisión de la bibliografía sólo hemos podido encontrar la publicación de medio centenar de casos que fueron diagnosticados en mayores de 18 años.
Por ello, se ha considerado de interés la presentación de 4 personas adultas que han sido diagnosticadas de hipoplasia pulmonar en nuestro servicio de neumología, junto a una revisión de la literatura.

\section{CASOS APORTADOS}

Caso 1: Varón de 20 años de edad que consultó por presentar tos, expectoración hemática y dolor torácico izquierdo. A la auscultación se apreciaba disminución del murmullo vesicular en ese hemitórax. En la radiografía de tórax aparecía una pérdida de volumen de hemitórax izquierdo (desviación del mediastino hacia la izquierda, eleva-

Trabajo aceptado: 25 de enero de 2001

Correspondencia: Francisco Carrión Valero. Servicio de Neumología. Hospital Clínico Universitario. Avda Blasco Ibáñez, 17. 46010 Valencia (España). 
ción diafragmática e hiperinsuflación del hemitórax derecho). El electrocardiograma era normal. En el ecocardiograma había dilatación de la aurícula y ventrículo derechos, con presión en arteria pulmonar $39 \mathrm{~mm} \mathrm{Hg}$. La arteriografía puso de manifiesto una arteria pulmonar izquierda de pequeño calibre con hipoplasia del árbol vascular. Posteriormente, el paciente presentó dos nuevos episodios de hemoptisis franca en el contexto de infecciones de vías respiratorias, el último a los 39 años de edad, evolucionando satisfactoriamente con reposo y antitusígenos. En la actualidad tiene 40 años y se encuentra asintomático.

Caso 2: Varón de 24 años con antecedente de síndrome de Down, que ingresa por presentar tos y disnea. En la auscultación se apreciaba la abolición del murmullo vesicular en base izquierda. En la radiografía de tórax existía pérdida de volumen del hemitórax izquierdo, con elevación del hemidiafragma y desplazamiento mediastínico ipsilateral, así como un quiste aéreo en lóbulo superior izquierdo y bronquiectasias basales izquierdas. El electrocardiograma y ecocardiograma eran normales. Broncoscopia: mucosa bronquial inflamatoria en el lóbulo inferior izquierdo; en el lóbulo superior derecho los bronquios están poco desarrollados. La TC torácica confirmó el diagnóstico de hipoplasia pulmonar. Cariotipo: trisomía 21. En la evolución posterior ha presentado diversos episodios de infección de vías respiratorias que mejoraron con tratamiento antibiótico ambulatorio. En la actualidad tiene 46 años y se encuentra bien.

Caso 3: Mujer de 50 años que consultó por presentar desde un año antes episodios repetidos de tos, disnea y sibilantes. En la radiografía de tórax se apreció una pérdida de volumen del hemitórax izquierdo, con imágenes quísticas, así como hiperinsuflación del pulmón derecho. En la exploración física presentaba semiología de broncoespasmo y disminución del murmullo vesicular en la base del pulmón izquierdo. La TC torácica puso de manifiesto una pérdida de volumen de pulmón izquierdo con múltiples bronquiectasias quísticas y varicosas y disminución del calibre vascular, sobre todo de la arteria pulmonar (Fig. 1). Electrocardiograma y ecocardiograma normales. Cariotipo normal. Con broncodilatadores ha evolucionado favorablemente.

Caso 4: Varón de 30 años de edad que consultó por presentar disnea de reposo y ortopnea en el contexto de una infección de vías respiratorias. A la auscultación aparecía un soplo sistólico en foco mitral así como disminución del murmullo vesicular en el hemitórax izquierdo y estertores crepitantes en ambas bases pulmonares. La radiografía de tórax puso de manifiesto una atelectasia del pulmón izquierdo, con hiperinsuflación del pulmón contralateral (Fig. 2). Electrocardiograma: arritmia completa por fibrilación auricular e hipertrofia ventricular derecha. El ecocardiograma demostró una insuficiencia mitral, con aurícula izquierda dilatada e hipertrofia del ventrículo derecho. En la broncoscopia destacaba la existencia de un árbol bronquial izquierdo con yemas rudimentarias de los bronquios segmentarios. Con digital y diuréticos evolucionó favorablemente. En la actualidad tiene 50 años y se encuentra estable.

En la tabla I están resumidos los datos clínicos de los pacientes, así como los resultados de las pruebas funcionales respiratorias.

\section{DISCUSIÓN}

La hipoplasia pulmonar es una rara anomalía congénita del desarrollo pulmonar en la que existe una disminución en el número o tamaño de las vías aéreas, alvéolos o vasos pulmonares, aunque la morfología grosera del pulmón es normal (6). La hipoplasia pulmonar puede ser primaria o secundaria. En el primer caso no existe patología coexistente y la mayoría de los pacientes mueren de forma precoz. Por el contrario, en la hipoplasia pulmonar secundaria se ha descrito un amplio espectro de anomalías asociadas que posiblemente están implicadas en su patogenia (Tabla II).

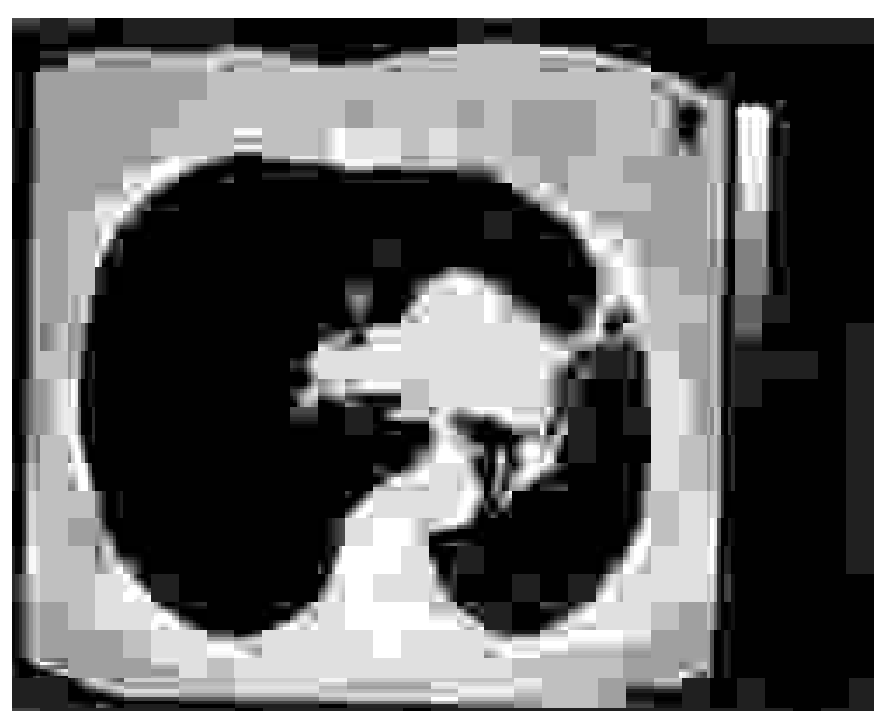

Fig. 1. Tc torácica (caso 3): pérdida de volumen de pulmón izquierdo.

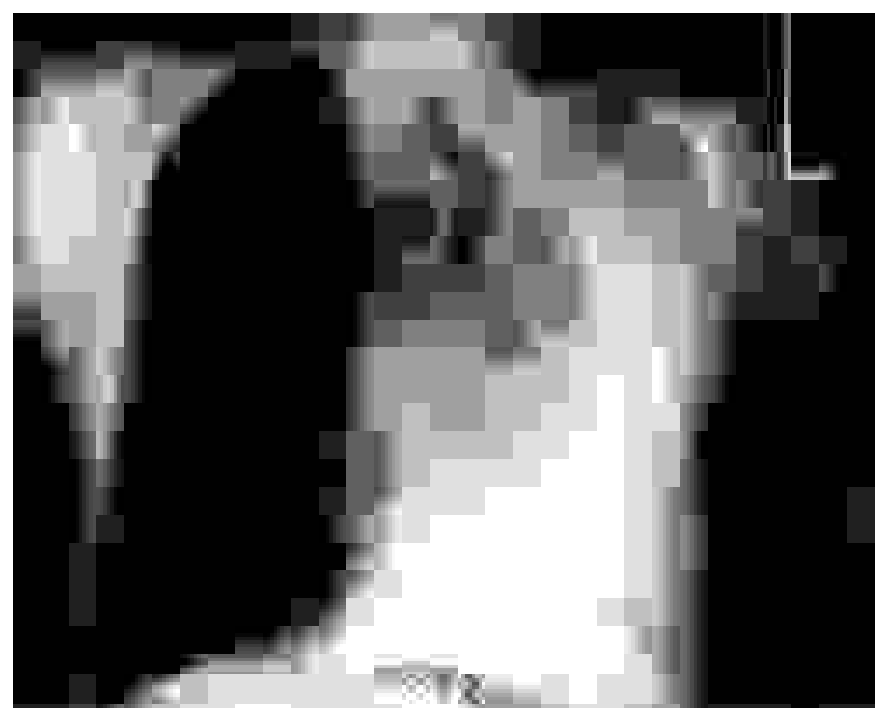

Fig. 2. Rx tórax (caso 4): atelectasia pulmón izquierdo.

En modelos animales de experimentación se ha demostrado que en la vida intrauterina, tanto la injuria medular cervical (7) como la sección bilateral del nervio frénico (8) inducen el desarrollo de una la hipoplasia pulmonar. De esta forma, el sistema nervioso central, al mantener los movimientos respiratorios normales en el feto, parece jugar un importante papel en el desarrollo pulmonar. Por ello, se ha especulado que anomalías no evidentes en el control del sistema nervioso central pudieran estar implicadas en la patogenia de la hipoplasia pulmonar primaria.

En el caso de la hipoplasia pulmonar secundaria, un espacio torácico insuficiente (hernia diafragmática, polihidramnios), enfermedades neuromusculares o neurológicas (anencefalia) o la agenesia renal pueden ocasionar la hipoplasia $(9,10)$. Además, es probable que en algún caso existan defec- 
TABLA I

SERIE ACTUAL: DATOS CLÍNICOS Y DE EXPLORACIÓ N FUNCIONAL RESPIRATO RA

\begin{tabular}{|c|c|c|c|c|}
\hline & Casol & Caso II & Caso III & Caso IV \\
\hline Edad de diagnóstico & 20 & 24 & 50 & 30 \\
\hline Localización & Izquierda & Izquierda & Izquierda & Izquierda \\
\hline Sintomatología inicial & $\begin{array}{l}\text { Dolor torácico, } \\
\text { tos, hemoptisis }\end{array}$ & $\begin{array}{l}\text { Bronquitis } \\
\text { recidivante, } \\
\text { disnea }\end{array}$ & Broncoespasmo & $\begin{array}{l}\text { Disnea, } \\
\text { insuficiencia } \\
\text { cardiaca }\end{array}$ \\
\hline $\begin{array}{l}\text { Anomalías } \\
\text { asociadas }\end{array}$ & Ninguna & $\begin{array}{l}\text { Síndrome de } \\
\text { Down, } \\
\text { bronquiectasias }\end{array}$ & Bronquiectasias & $\begin{array}{l}\text { Insuficiencia } \\
\text { mitral }\end{array}$ \\
\hline FVC $(\%)$ & 94 & 36 & 77 & 47 \\
\hline $\mathrm{FEV}^{1}(\%)$ & 80 & 42 & 76 & 35 \\
\hline $\mathrm{FEV}^{1} / \mathrm{FVC}(\%)$ & 80 & 100 & 84 & 61 \\
\hline $\mathrm{PaO}_{2}(\mathrm{mmHg})$ & 92 & 61 & 91 & 59 \\
\hline $\mathrm{PaCO}_{2}(\mathrm{mmHg})$ & 38 & 45 & 36 & 42 \\
\hline Técnica de diagnóstico & Arteriografía & TC & $\mathrm{TC}$ & Broncoscopia \\
\hline Sexo & Hombre & Hombre & M ujer & Hombre \\
\hline
\end{tabular}

FEV ${ }^{1}$ : volumen espiratorio máximo en el ler segundo. FVC: capacidad vital forzada.

TABLA II

ANOM ALÍAS ASO CIADAS EN LA HIPO PLASIA PULMONAR
SECUNDARIA

Anomalías genitourinarias:

Síndrome de Potter (agnesia renal,
hipoplasia pulmonar y facies
característica
Cifoescoliosis
Ebstein $^{14}, \mathrm{CIA}^{11}$, DAP

\section{Anomalías óseas:}

Cardiopatías:

Síndrome de Down ${ }^{12}$

Agnesia diafragmática ${ }^{5}$

Síndrome de Klippel-Feil

Isoinmunización rhesus

Arteria pulmonar izquierda aberrante ${ }^{3}$

Hernia diafragmática

CIA: Comunicación ínterauricular

DAP: Ductus arterioso persistente

tos bioquímicos y metabólicos, como una disminución en la síntesis de prolina (11).

Por otro lado, se ha registrado la presencia de hipoplasia pulmonar en casos esporádicos de síndrome de Down (12), igual que en el caso número II de nuestra serie, y en otras cromosomopatías (13): delección de los brazos largos del cromosoma 13, trisomía parcial 7q y trisomías 13 y 18 . En el síndrome de Down, un estudio histopatológico realizado por Tarroch et al (12) demostró que la hipoplasia pulmonar se caracteriza por una hipoalveolización. El recuento alveolar demostró 4,6 alvéolos por acino, con espacios aéreos anormalmente grandes, al contrario que sucede en otros tipos de hipoplasia pulmonar en los que existe una disminución del tamaño alveolar (14).

El desarrollo embriológico del pulmón se inicia a los 24 días de gestación, a partir de una invaginación del intestino anterior, y evoluciona en distintos estadios: embrionario (has- ta la semana 8), pseudoglandular (hasta la semana 16), canalicular, sacular y alveolar (10). Cualquier factor que interfiera el normal desarrollo ocasionará diferentes malformaciones que generalmente se manifiestan en la infancia. Cuando la agresión acontece durante el estadio pseudoglandular ocasiona una hipoplasia pulmonar, con reducción de las divisiones bronquiales, la complejidad acinar y la vascularización pulmonar, tal como se observa en los pacientes de la serie actual.

En la literatura existen múltiples casos de hipoplasia pulmonar en neonatos (15). Sin embargo, el diagnóstico en personas mayores de 18 años, como en los cuatro pacientes de la serie actual, es un hallazgo extraordinariamente raro. De hecho, en una reciente revisión bibliográfica, Comet et al (1) sólo pudieron documentar 48 casos de hipoplasia pulmonar diagnosticada en el adulto. Los autores comunicaron una serie de 5 pacientes en los que la edad de presentación oscilaba entre los 45 y 67 años, mayor que en los pacientes de la serie actual (20-50 años).

En los cuatro pacientes de nuestra serie estaba afectado el pulmón izquierdo. En 35 de los 48 casos antes citados (73\%) (1) también estaba afectado el pulmón izquierdo, aunque no se conoce una razón que explique esta predilección.

En 3 de los 4 pacientes actuales encontramos malformaciones asociadas. En el caso número IV, la valvulopatía mitral fue responsable de la sintomatología inicial y permitió el diagnóstico de la malformación congénita. Por otro lado, debido a la producción y retención de secreciones en el tejido pulmonar rudimentario, la hipoplasia pulmonar predispone a infecciones respiratorias de repetición (16). De este modo, las bronquiectasias que presentaban los pacientes II y III podrían ser causa o consecuencia de dichas infecciones.

Desde el punto de vista fisiopatológico, a pesar de que los pacientes suelen mostrar un patrón ventilatorio restrictivo en la espirometría (17), la infección recidivante puede facilitar el desarrollo de una obstrucción ventilatoria, tal como sucedió en el caso número IV de la serie actual.

Por otro lado, la posibilidad de un diagnóstico antenatal de los pacientes ha suscitado interés en la literatura. Sin embargo, ninguno de los índices propuestos ha mostrado una sensi- 
bilidad y especificidad suficientes para permitir la toma de decisiones clínicas.

La TC torácica fue la exploración que permitió el diagnóstico en los casos II y III. Por el contrario, en los pacientes I y IV una arteriografía pulmonar y una broncoscopia fueron las exploraciones que hace 20 años facilitaron el diagnóstico de esta alteración en el desarrollo pulmonar. Aunque de una manera preliminar, estos dos casos ya habían sido comunicados previamente (18). Sin embargo, debido a que no fueron recogidos en la revisión bibliográfica de Comet et al (1), nos ha parecido oportuno incorporarlos a la serie actual, y describir su evolución en este periodo con tratamiento conservador.

En definitiva, la existencia de series relativamente amplias como la actual, pone de manifiesto que la hipoplasia pulmonar en el adulto puede ser más frecuente de lo que se ha comunicado hasta ahora. La TC torácica rutinaria cuando la radiografía lo sugiera debería facilitar el diagnóstico y permitir la prevención y tratamiento precoz de las infecciones respiratorias y las anomalías congénitas asociadas, mejorando el pronóstico de los enfermos.

\section{Bibliografía}

1. Comet R, Mirapeix RM, Marín A, Castañer E, Sans J, Domingo C. Hipoplasia pulmonar en el adulto: embriología, presentación clínica y métodos diagnósticos. Experiencia propia y revisión de la literatura. Arch. Bronconeumol 1998; 34 (1): 48-51.

2. Nakamura Y, Harada K, Yumamoto I, Vemura Y, Okamoto K, Fukuda $\mathrm{S}$, et al. Human pulmonary hipoplasia. Statiscal, morphological and biochemical study. Arch Pathol Lab Med. 1992; 116: 635-42.

3. Salmerón I, Fernández F, Bustos A, Ferreiros J, Arrazola J, Fernández JM. Arteria pulmonar izquierda aberrante asociada a hipoplasia pulmonar derecha. Radiología 1995; 37 (5): 358-61.

4. Durán M, Gómez I, Palacio A. Anomalía de Ebstein con hipoplasia pulmonar. Diagnostico mediante ecocardiografía Doppler color en el feto. Rev Esp Cardiol 1992; 5: 541-2.

5. Sousa R, Trigo J, Bascuas M, Gutierrez M, Bueno A, Bascuas JL. Agenesia diafragmática asociada a otras malformaciones. Acta Pediatr Esp 1987; 45 (8): 462-4.

6. Green AR, Shaw DG, Haworth SH. Familial pulmonary hypoplasia: plain film appearances with histopathological correlation. Pediatr. Radiol, 1999; 29: 455-8.

7. Liggins GC, Vilos GA, Campos GA, Kitterman JA, Lee CH. The effect off spinal cord transection on lung development in fetal sheep. J Develop Physiol 1981; 3: 267-74.

8. Fewell JE, Lee CC, Kitterman JA. Effects of phrenic nerve section on the respiratory system on fetal lambs. J Appl Physiol 1981; 51: 293 -

9. Fraser RG, Paré JA, Paré PD. Diagnóstico de las enfermedades del tórax. $3^{a}$ Ed. 1992. Ed Panamericana: 661-731.

10. Michele R, Lauria MD, Bernard, Gonik MD,. Pulmonary hypoplasia: pathogenesis, diagnosis, and antenatal prediction. Obstet.Gynecol 1995; 86: 466-75.

11. Pellicer C, Menéndez R, Perpiñá M, Meseguer A, Fombellida JC, Marco V. Anomalías congénitas del pulmón: agenesia, aplasia e hipoplasia. Arch. Bronconeumol. 1983; 19 (4): 141-7.

12. Tarroch X, Rovirosa N, Torán N, Lozano C. Hipoplasia e hipertensión pulmonar en un síndrome de Down: valoración pronostica mediante la biopsia pulmonar. Rev Esp Cardiol 1989; 42: 348-50.

13. Fontaiña M, Solar A, Pereira S, Goyanes V, Sirvent J, Rodríguez G. Trisomía parcial 7q (7q22 -> 7qter) e hipoplasia pulmonar bilateral. Rev Esp Pediatr 1993; 49 (3): 265-7.

14. Bernal F, Bravo JL, Rami R, Aroca M, Alix A, Lahoz F, et al. Hipoplasia pulmonar. A propósito de un caso. Rev.Clin.Esp 1984; 173: 57-60.

15. Vizcaíno A, Salmerón JR. Agenesia e hipoplasia pulmonar. Estudio clínico de 20 casos. Bol.Med.Hosp.Inf.Mex. 1974; 35 (5): 899-916.

16. Arteche MD, Oleaga, Agote A, Echeberria A, Vega-Hazas G. Malformaciones congénitas bronquiales. Radiología 1989; 31: 289-95.

17. Báguena R, Marín J, Calpe L, Servera E, Pérez D, Domínguez A. Las hipoplasias pulmonares. Med Esp 1985; 84: 17-22.

18. Marín J, Calpe JL, Servera E, Baguena R. Tres casos de hipoplasia pulmonar en la edad adulta. Med. Esp. 1985; 84: 53-56. 